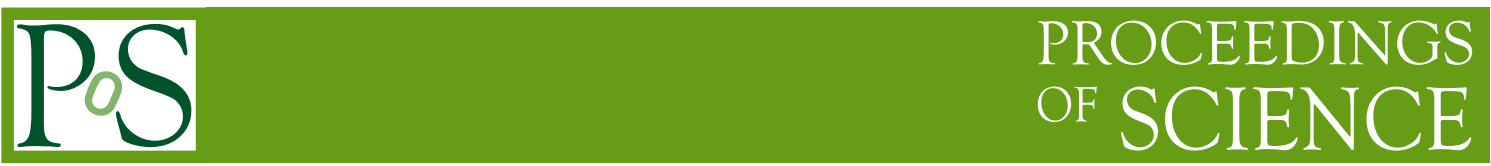

\title{
Recent Results on Soft QCD Topics from ATLAS
}

\author{
Andrey Minaenko, on behalf of the ATLAS Collaboration \\ Institute for High Energy Physics, Protvino, Russia \\ E-mail: Andrey.Minaenko@cern.ch
}

\begin{abstract}
The ATLAS collaboration has performed several measurements in special data sets with low LHC beam currents, recorded at a center-of-mass energy of $13 \mathrm{TeV}$ : Measurements of the inclusive charged-particle multiplicity and its dependence on transverse momentum and pseudorapidity are presented and compared with predictions of various MC generators. The collaboration has also performed measurements of the number and transverse-momentum sum of charged particles as a function of properties of the leading high $p_{T}$ track in the event at a center-of-mass energy of $13 \mathrm{TeV}$. The results are compared to predictions of several MC generators. In addition, the total inelastic proton-proton cross section and the diffractive part of the inelastic cross section was measured, using special forward scintillators of the calorimeters. The latter result completes the measurement of the elastic pp cross section in a dedicated run with high beta* optics at $8 \mathrm{TeV}$ centre-of-mass energy with the ALFA Roman Pot detector. From the extrapolation of the differential elastic cross section to $t=0$, using the optical theorem, the total cross section is extracted with the luminosity-dependent method with high precision. Furthermore, the nuclear slope of the elastic t-spectrum and the total elastic and inelastic cross sections are determined. Finally, the collaboration has studied the hard double parton interactions (DPI) in events with 4 hadronic jets and translated into a measurement of the effective DPI cross section. Several DPI-sensitive variables are unfolded to particle level and compared to predictions of different MC models.
\end{abstract}

55th International Winter Meeting on Nuclear Physics

23-27 January, 2017

Bormio, Italy 


\section{Introduction}

The ATLAS multi-purpose particle detector is described in [1]. Recent ATLAS results on soft QCD topics are present in this report. They provide insight into the strong interaction in the lowenergy, non-perturbative region of quantum chromodynamics (QCD). Particle interactions at these energy scales are typically described by QCD-inspired models implemented in Monte Carlo (MC) event generators with free parameters that can be constrained by such measurements. The studies give also better knowledge of background processes for new physics signals.

Four results are presented: i) charged-particle distributions in minimum bias pp interactions at $\sqrt{s}=13 \mathrm{TeV}$ [2]; ii) measurement of charged-particle distributions sensitive to the underlying event in $\sqrt{s}=13 \mathrm{TeV}$ pp collisions [3]; iii) measurement of the total cross section from elastic scattering in pp collisions at $\sqrt{s}=8 \mathrm{TeV}$ [4]; iv) study of hard double-parton scattering in four-jet events in pp collisions at $\sqrt{s}=7 \mathrm{TeV}[5]$.

\section{Charged-particle distributions in $\sqrt{s}=13 \mathrm{TeV}$ pp interactions}

This study [2] presents inclusive measurements of primary charged-particle distributions in $\mathrm{pp}$ collisions at a centre-of-mass energy of $\sqrt{s}=13 \mathrm{TeV}$, using data recorded by the ATLAS experiment at the LHC corresponding to an integrated luminosity of approximately $170 \mu \mathrm{b}^{-1}$.

All primary charged particles are required to have a momentum component transverse to the beam direction, $p_{T}$, of at least $500 \mathrm{MeV}$ and absolute pseudorapidity, $|\eta|$, less than 2.5 . The mean charged particle densities as functions of $\eta$ and $p_{T}$ are measured as well as the mean $p_{T}$ of all primary charged particles versus the number of primary charged particles in an event, $n_{c h}$.

The PYTHIA8, EPOS, and QGSJET-II MC generators are used to correct the data for detector effects and to compare with particle-level corrected data. In PYTHIA8 inclusive hadron-hadron interactions are described by a model that splits the total inelastic cross section into non-diffractive (ND) processes, dominated by t-channel gluon exchange, and diffractive processes involving a colour-singlet exchange. The simulation of ND processes includes multiple parton-parton interactions (MPI). EPOS provides an implementation of a parton-based Gribov-Regge theory, which is an effective QCD-inspired field theory describing hard and soft scattering simultaneously. QGSJET-II provides a phenomenological treatment of hadronic and nuclear interactions in the Reggeon field theory framework.

The data were recorded during a period with a special configuration of the LHC with low beam currents and reduced beam focusing, and thus giving a low expected mean number of interactions per bunch crossing $(\sim 0.005)$. Events were selected from colliding proton bunches using a trigger which required one or more minimum-bias trigger scintillator counters above threshold on either side of the detector.

The data are corrected to obtain inclusive spectra for primary charged particles satisfying the particle-level kinematic requirements. These corrections account for inefficiencies due to trigger selection, vertex and track reconstruction. The primary track reconstruction efficiency, $\varepsilon_{t r k}$, is determined from the simulation, corrected to account for differences between data and simulation in the amount of detector material between the pixel and SCT detectors. The efficiency is parameterised in two-dimensional bins of $p_{T}$ and $\eta$. The track reconstruction efficiency depends on the 
amount of material in the detector, due to particle interactions that lead to efficiency losses. The material within the ID was studied extensively during Run 1 and the amount of material was known to within $\pm 5 \%$. This gives rise to a systematic uncertainty in the track reconstruction efficiency of $\pm 0.6 \%( \pm 1.2 \%)$ in the most central (forward) region. For the $p_{T}$ distribution a Bayesian unfolding is applied to correct the measured track $p_{T}$ distribution to that for primary particles.

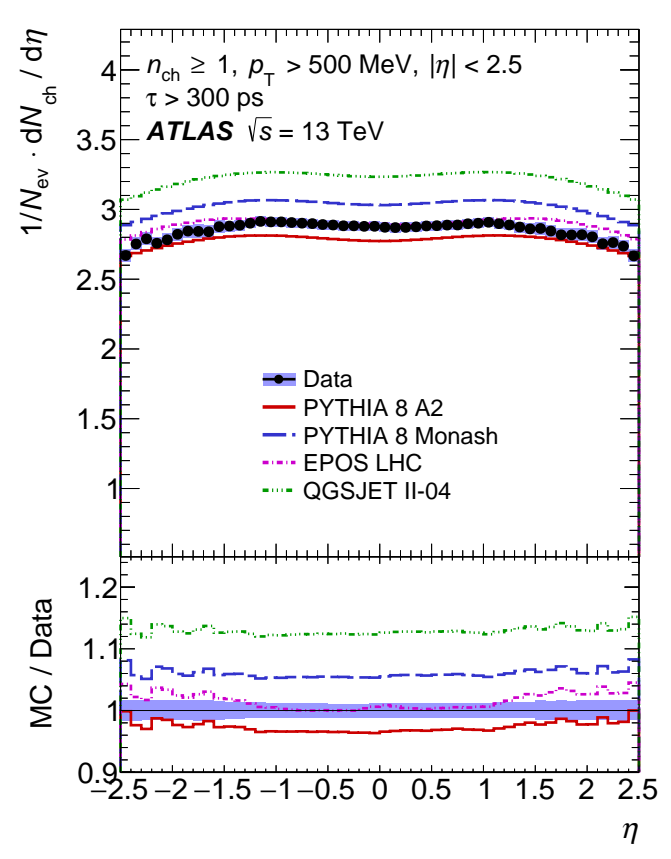

(a)

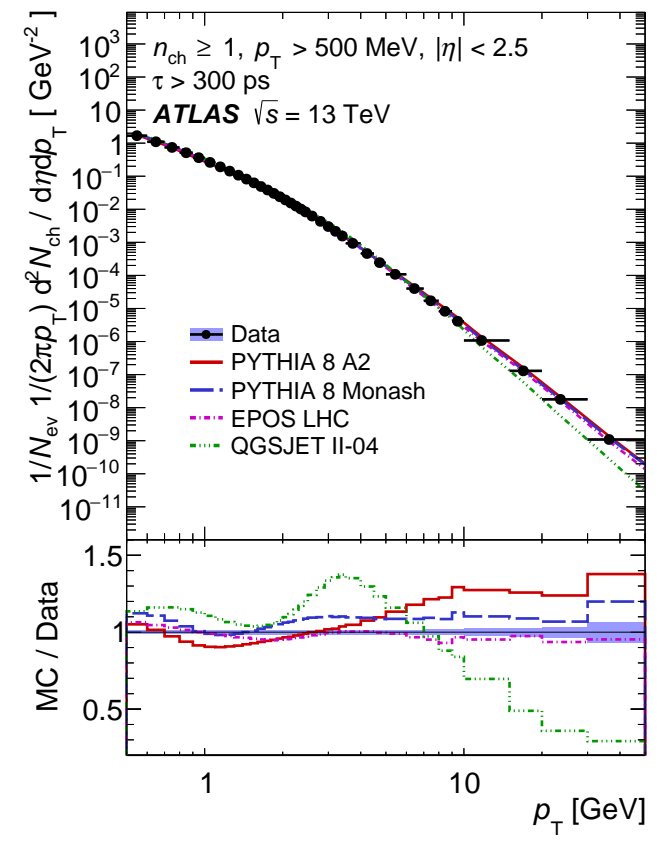

(b)

Figure 1: Primary-charged-particle multiplicities as a function of (a) pseudorapidity, $\eta$, and (b) transverse momentum, $p_{\mathrm{T}}$ in events with $n_{c h} \geq 1, p_{T}>500 \mathrm{MeV}$ and $|\eta|<2.5$. The dots represent the data and the curves the predictions from different MC models [2].

The corrected distributions for primary charged particles are shown in Figure 1. Figure 1a shows the multiplicity of charged particles as a function of pseudorapidity. The mean particle density is roughly constant at 2.9 for $|\eta|<1.0$ and decreases at higher values of $|\eta|$. EPOS describes the data for $|\eta|<1.0$, and predicts a slightly larger multiplicity at larger $|\eta|$ values. QGSJET-II and PYTHIA8 Monash predict multiplicities that are too large by approximately $15 \%$ and $5 \%$ respectively. PYTHIA8 A2 predicts a multiplicity that is $3 \%$ too low in the central region, but describes the data well in the forward region.

Figure $1 \mathrm{~b}$ shows the charged-particle transverse momentum distribution. EPOS describes the data well over the entire $p_{T}$ spectrum. The PYTHIA8 tunes describe the data reasonably well, but are slightly above the data in the high- $p_{T}$ region. QGSJET-II gives a poor prediction over the entire spectrum, overshooting the data in the low- $p_{T}$ region and undershooting it in the high- $p_{T}$ region.

The mean number of primary charged particles in the central region is compared to previous measurements at different $\sqrt{s}$ values in Figure 2 together with the MC predictions. The mean number of primary charged particles increases by a factor of 2.2 when $\sqrt{s}$ increases by a factor of 
about 14 from $0.9 \mathrm{TeV}$ to $13 \mathrm{TeV}$. EPOS and PYTHIA8 A2 describe the dependence on $\sqrt{s}$ very well, while PYTHIA8 Monash and QGSJET-II predict a steeper rise in multiplicity with $\sqrt{s}$.

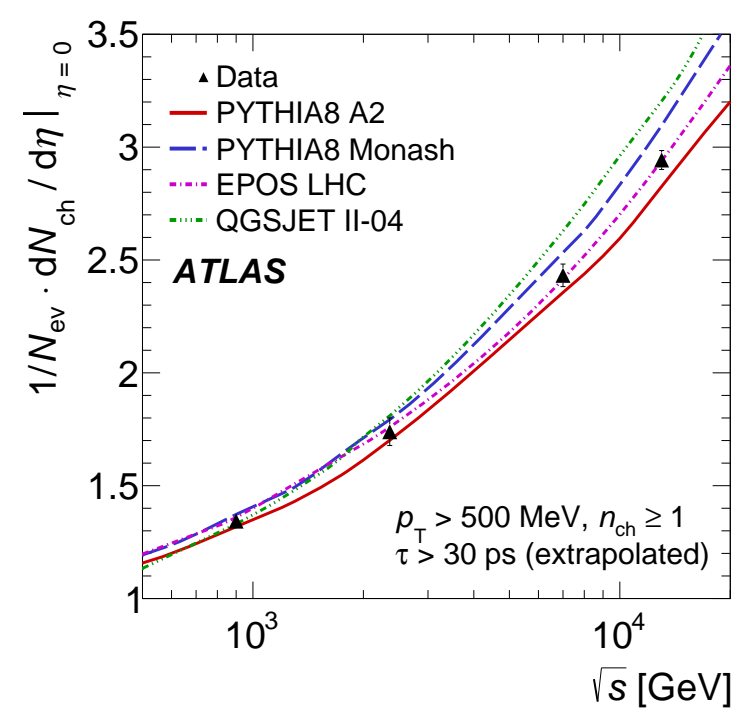

Figure 2: The average primary-charged-particle multiplicity in pp interactions per unit of pseudorapidity, $\eta$, for $|\eta|<0.2$ as a function of the centre-of-mass energy. The data are compared to various particle-level MC predictions [2].

\section{Measurement of charged-particle distributions sensitive to the underlying event in $\sqrt{s}=13 \mathrm{TeV}$ pp collisions}

It is important to have a good understanding not only of the primary short-distance hard scattering process, but also of the accompanying interactions of the rest of the pp collision - collectively termed the underlying event (UE). The UE can receive contributions from the QCD evolution of colour connections between the hard scattering and the beam-proton remnants, and from additional hard scatters in the same pp collision, termed multiple partonic interactions (MPI). As it is significantly influenced by physics not currently calculable from first principles, the measurement of the UE's properties is crucial not only for better understanding of the mechanisms involved but also to provide input for empirical tuning of the free parameters of phenomenological UE models in MC event generators.

This measurement uses the established form of UE observables, in which the azimuthal plane of the event is segmented into several distinct regions with differing sensitivities to the UE. As illustrated in Figure 3, the azimuthal angular difference with respect to the leading (highest- $p_{T}$ ) charged particle, $|\Delta \phi|=\left|\phi-\phi_{\text {lead }}\right|$, is used to define the corresponding regions. The towards and away regions are dominated by particle production from the hard process. In contrast, the transverse region is more sensitive to the UE, and observables defined inside it are the primary focus of UE measurements. A further refinement is to distinguish on a per-event basis between the more and the less active sides of the transverse region, defined in terms of their relative scalar sums of primary charged-particle $p_{T}$ and termed "trans-max" and "trans-min" respectively. The trans-min region is 


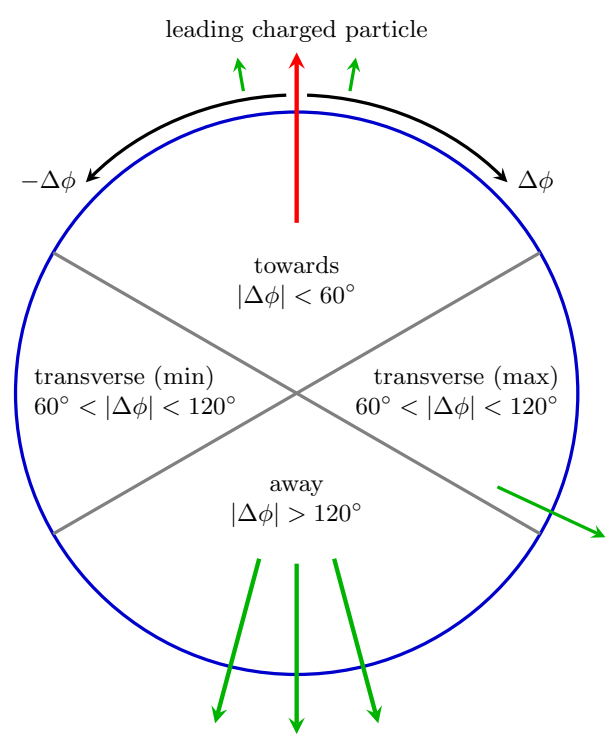

Figure 3: Definition of regions in the azimuthal angle with respect to the leading (highest- $p_{T}$ ) charged particle, with arrows representing particles associated with the hard scattering process and the leading charged particle highlighted in red. Conceptually, the presence of a hard-scatter particle on the right-hand side of the transverse region, increasing its $\Sigma p_{T}$, typically leads to that side being identified as the "trans-max" and hence the left-hand side as the "trans-min", with maximum sensitivity to the UE.

relatively insensitive to wide-angle emissions from the hard process, and the difference between trans-max and trans-min observables hence represents the effects of hard-process contamination.

In order to compare the measured underlying-event distributions with model predictions in the particle-level fiducial phase space, the observables have been corrected for detector effects. These corrections include explicit accounting for inefficiencies due to the trigger selection, vertexing, and track reconstruction, by weighting the events and tracks by inverse efficiencies. The event selection and correction procedures are similar to those described in the previous section.

Figure 4 focuses on the UE-dominated trans-min region. The trans-min is the most sensitive to MPI effects, i.e. the pedestal, while the trans-max includes both MPI and hard-process contamination. The comparisons to MC models are made in these plots. There is significant variation in performance between the models, with PYTHIA8 Monash tune and HERWIG7 giving the best description of data in the plateau region of trans-min, followed within $10 \%$ by the other PYTHIA 8 tunes. EPOS, however, slightly overestimates in the "ramp" region to the pedestal effect plateau, and on the plateau it underestimates the pedestal height by around 20\%. HERWIG7 and PYTHIA8 A2 both mismodel the transition, with a severe undershoot for HERWIG below $p_{T}^{\text {lead }}$ of $5 \mathrm{GeV}$, and a milder but broader undershoot from A2 which extends up to $p_{T}^{\text {lead }}$ of about $20 \mathrm{GeV}$.

\section{Measurement of the total cross section from elastic scattering in pp collisions at $\sqrt{s}=\mathbf{8} \mathbf{~ T e V}$}

The total cross section for pp interactions characterizes a fundamental process of the strong 


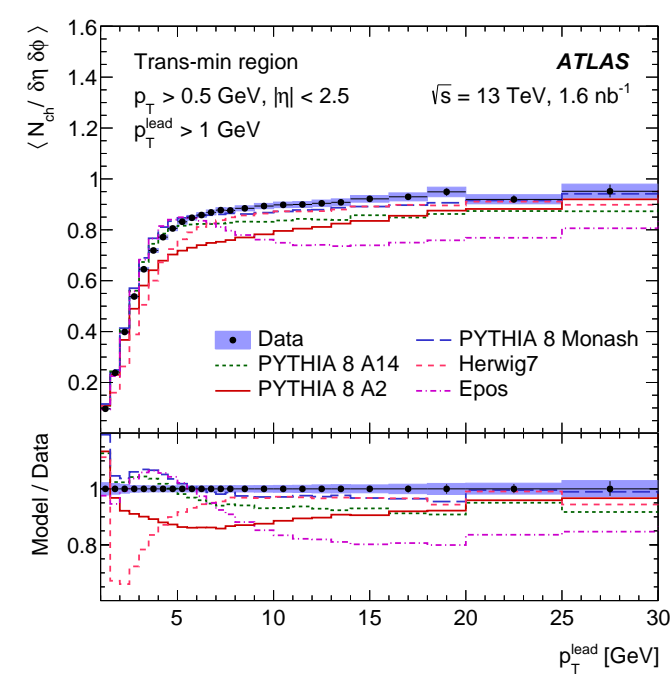

(a)

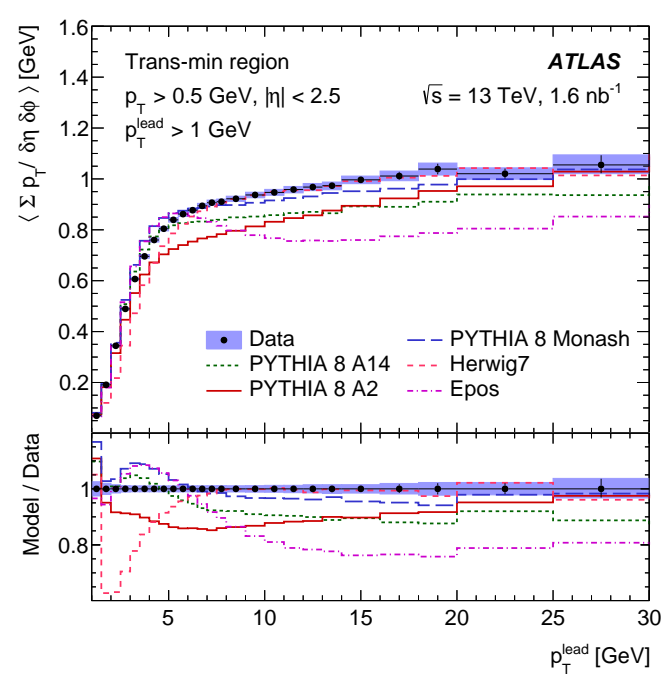

(b)

Figure 4: Mean densities of charged-particle multiplicity $N_{c h}$ (left) and $\Sigma p_{T}$ (right) as a function of leading charged-particle $p_{T}$, in the trans-min region [3].

interaction. A measurement of the total pp cross section at $\sqrt{s}=8 \mathrm{TeV}$ is presented in [4]. An integrated luminosity of $500 \mu \mathrm{b}^{-1}$ was accumulated in a special run with high- $\beta^{*}$ beam optics to measure the differential elastic cross section as a function of the momentum transfer variable $t$. The total cross section $\sigma_{t o t}$ is measured via optical theorem: $\sigma_{t o t}=4 \pi \cdot \operatorname{Im}\left[f_{e l}(t \rightarrow 0)\right]$, relating $\sigma_{t o t}$ to the elastic-scattering amplitude extrapolated to the forward direction $f_{e l}(t \rightarrow 0)$.

The elastic-scattering data were recorded with the ALFA sub-detector [6]. It consists of Roman Pot (RP) tracking-detector stations placed at distances of $237 \mathrm{~m}$ and $241 \mathrm{~m}$ on either side of the ATLAS interaction point (IP). Each station houses two vertically moveable scintillating fibre detectors which are inserted in RPs and positioned close to the beam for data taking. The spatial resolution of the detectors is about $35 \mu \mathrm{m}$.

The four-momentum transfer is calculated from the scattering angle $\theta^{*}$ and the nominal beam momentum $p=3988 \pm 26 \mathrm{GeV}$. The scattering angle is calculated from the proton trajectories and beam optics parameters. To reconstruct the differential elastic cross section a number of corrections were applied to the measured t-distribution: background subtraction, acceptance correction, reconstruction efficiency, trigger efficiency, DAQ dead time correction, integrated luminosity factor, as well as the unfolding procedure.

The theoretical model to fit the elastic data consists of three terms: Nuclear, Coulomb and Coulomb-Nuclear Interference. The dominant Nuclear term in $\frac{d \sigma_{e l}}{d t}$ is

$$
\sigma_{\text {tot }}^{2} \frac{1+\rho^{2}}{16 \pi(\hbar c)^{2}} \cdot \exp (-B|t|)
$$

where $\rho=\operatorname{Re}\left(f_{e l}\right) / \operatorname{Im}\left(f_{e l}\right)=0.1362 \pm 0.0034$ and $B$ is the nuclear slope.

The resulting $\frac{d \sigma_{e l}}{d t}$ distribution is shown in Figure 5a together with the fitting curve including 


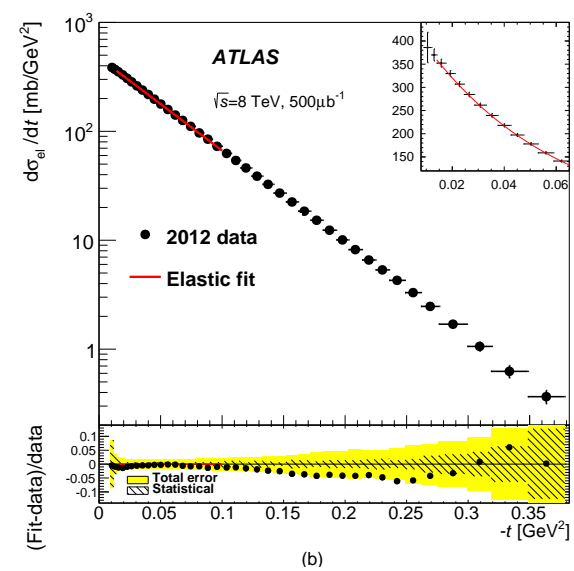

(a)

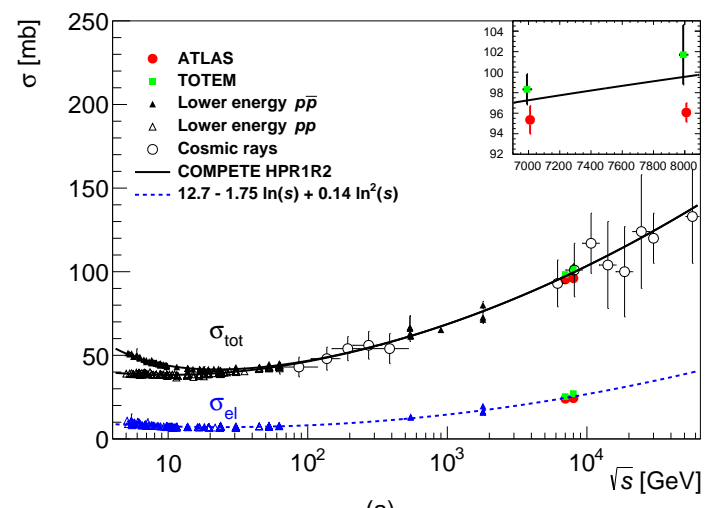

(b)

Figure 5: a) The fit of the theoretical prediction to the differential elastic cross section with $\sigma_{t o t}$ and $\mathrm{B}$ as free parameters. b) Comparison of total and elastic cross-section measurements presented here with other published measurements and model predictions as a function of the centre-of-mass energy [4].

all the three terms. The final results for $\sigma_{t o t}$ and $B$ are:

$$
\begin{aligned}
& \sigma_{\text {tot }}=96.07 \pm 0.18 \text { (stat.) } \pm 0.85 \text { (exp.) } \pm 0.31 \text { (extr.) } \mathrm{mb} \\
& B=19.74 \pm 0.05 \text { (stat.) } \pm 0.16 \text { (exp.) } \pm 0.15 \text { (extr.) } \mathrm{GeV}^{-2}
\end{aligned}
$$

The systematic and extrapolation errors are noticeably larger than the statistical ones. A number of systematic effects were taken in to account but the largest contribution is due to luminosity (1.5\%) and beam energy $(0.65 \%)$ uncertainties. The dominant extrapolation uncertainty is induced by the fit range variation.

\section{Study of hard double-parton scattering in four-jet events in pp collisions at $\sqrt{s}=\mathbf{7 ~ T e V}$}

In this study [5] inclusive four-jet events produced in pp collisions at a centre-of-mass of $\sqrt{s}=7 \mathrm{TeV}$ are analysed for the presence of hard double-parton scattering (DPS) using data corresponding to an integrated luminocity of $37.3 \mathrm{pb}^{-1}$ with $\langle\mu\rangle=0.41$. DPS is the simplest case of multi-parton interactions (MPI), which are rather abundant at the LHC energies and important for understanding of the full picture of hadronic collisions. Final four-jet state can be produced by a single parton scattering (SPS) ( $2 \rightarrow 4$ process) as well as by dijet DPS: $2 \mathrm{j}(\mathrm{A})$ and $2 \mathrm{j}$ (B), with A and $\mathrm{B}$ different, possibly overlapping, phase space regions. For this case a simplified formalism yields

$$
\sigma_{4 j}^{D P S}=f_{D P S} \cdot \sigma_{4 j}=\frac{1}{1+\delta_{A B}} \frac{\sigma_{2 j}^{A} \sigma_{2 j}^{B}}{\sigma_{e f f}}
$$

where, $\sigma_{2 j}^{A}$ and $\sigma_{2 j}^{B}$ are the cross sections for dijet events in the phase space regions A and B respectively, $\sigma_{4 j}\left(\sigma_{4 j}^{D P S}\right)$ is the inclusive (DPS) cross-section for four-jet events in the phase space 
region $A \oplus B, f_{D P S}$ represents the fraction of DPS events in the inclusive four-jet final state, the Kroneker $\delta_{A B}$ is equal to 1 , if $\mathrm{A}$ and $\mathrm{B}$ are the same. $\sigma_{\text {eff }}$ is the effective cross section, purely phenomenological parameter describing the effective overlap of the spatial distribution of partons in the plane perpendicular to the direction of motion. To extract $\sigma_{e f f}$, it is necessary therefore to measure three cross sections, $\sigma_{2 j}^{A}, \sigma_{2 j}^{B}$ and $\sigma_{4 j}$, and estimate $f_{D P S}$.

The DPS model contributes in two ways to the production of events with at least four jets, leading to two separate event classifications: i) the secondary scatter produces two of the four leading jets in the event and such events are classified as complete-DPS (cDPS); ii) three of the four leading jets are produced in the harder scatter, and the fourth jet is produced in the second scatter, such events are classified as semi-DPS (sDPS). The main challenge of the measurement is the extraction of $f_{D P S}=f_{c D P S}+f_{S D P S}$ from optimally selected measured observables.

Multi-jet events were MC generated using fixed-order QCD matrix elements $(2 \rightarrow n$, with $n=$ $2,3,4,5,6$ ) with Alpgen2.14, interfaced to Jimmy (MPI generation) and Herwig6.520 (hadronization). Event-record information was used to extract a sample of SPS candidate events. A sample of candidate DPS events was also extracted in order to study the topology of such events. Different but similar MC sample was used to derive the corrections for detector effects in all differential distributions in the data.

Jets in the data were identified using anti- $k_{t}$ algorithm with radius of $R=0.6$ and ordered in $p_{T}$. The measurement was performed using the dijet A sample and its two subsamples (dijet B and four-jet), selected using the following requirements:

$$
\begin{array}{llrl}
\text { Dijet A: } & N_{\text {jet }} \geq 2, \quad p_{T}^{1} \geq 20 \mathrm{GeV}, & p_{T}^{2} \geq 20 \mathrm{GeV}, & \left|\eta_{1,2}\right| \leq 4.4, \\
\text { Dijet B: } & N_{\text {jet }} \geq 2, \quad p_{T}^{1} \geq 42.5 \mathrm{GeV}, & p_{T}^{2} \geq 20 \mathrm{GeV}, & \left|\eta_{1,2}\right| \leq 4.4, \\
\text { Four-jet: } & N_{\text {jet }} \geq 4, \quad p_{T}^{1} \geq 42.5 \mathrm{GeV}, & p_{T}^{2,3,4} \geq 20 \mathrm{GeV}, & \left|\eta_{1,2,3,4}\right| \leq 4.4 .
\end{array}
$$

To estimate the DPS contribution to the four-jet data sample the usual approach is adopted: to fit the distribution of variables sensitive to cDPS and sDPS in the data to a combination of templates for the expected SPS, cDPS and sDPS contributions. The template for the SPS contribution is extracted from the MC sample, while the cDPS and sDPS templates are obtained by overlaying two dijet events from the data.

An artificial neural network (NN) is used for the classification of events, using as input 21 various variables, which describe the topology of a four-jet event. The output of the NN consists of three variables, which are interpreted as the probability for an event to be more like SPS $\left(\xi_{S P S}\right), \operatorname{cDPS}\left(\xi_{c D P S}\right)$ or sDPS $\left(\xi_{s D P S}\right)$, with $\xi_{S P S}+\xi_{c D P S}+\xi_{s D P S}=1$. As two of the variables are independent, the result can be presented for each an event as a point in a 2D plane (ternary plots, Figure 6). Figures $6 a, 6 b$ and $6 c$ show the NN output distributions for the pure test samples, SPS, cDPS and sDPS, presenting the separation power of the NN. The samples populate different but overlapping regions on the plane. The NN output in the data (Figure 6d) is visually consistent with a superposition of the three components.

To estimate the cDPS and sDPS fractions in four-jet events the ternary distribution in data $(\mathscr{D})$ is fitted to a weighted sum of the ternary distributions in the SPS $\left(\mathscr{M}_{S P S}\right)$, cDPS $\left(\mathscr{M}_{c D P S}\right)$ and $\operatorname{sDPS}\left(\mathscr{M}_{S D P S}\right)$ samples, each normalized to the measured four-jet cross-section in data, with the 


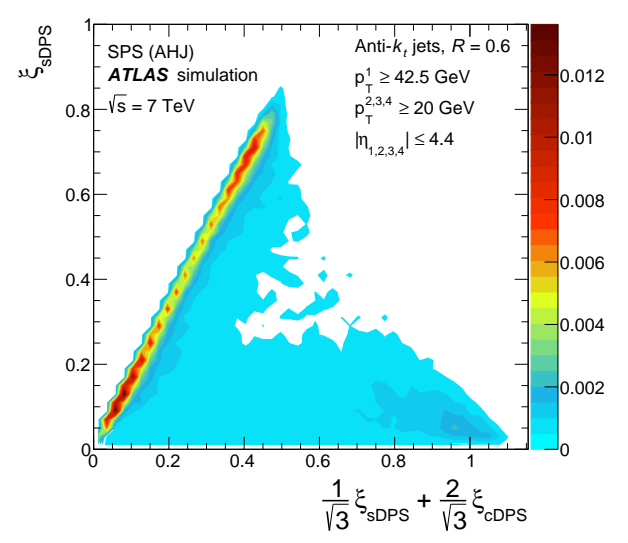

(a)

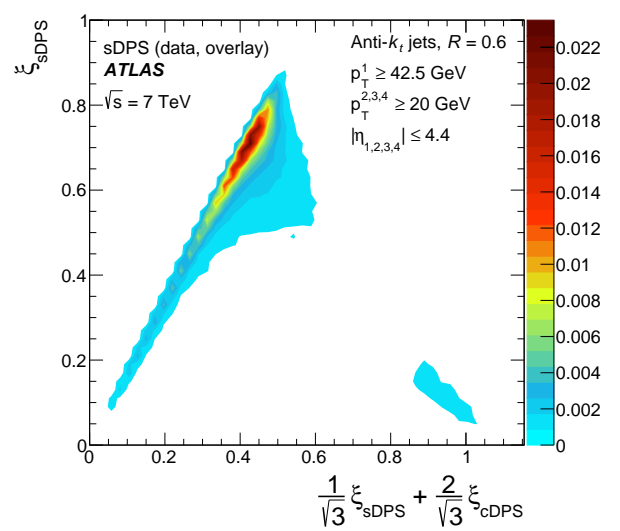

(c)

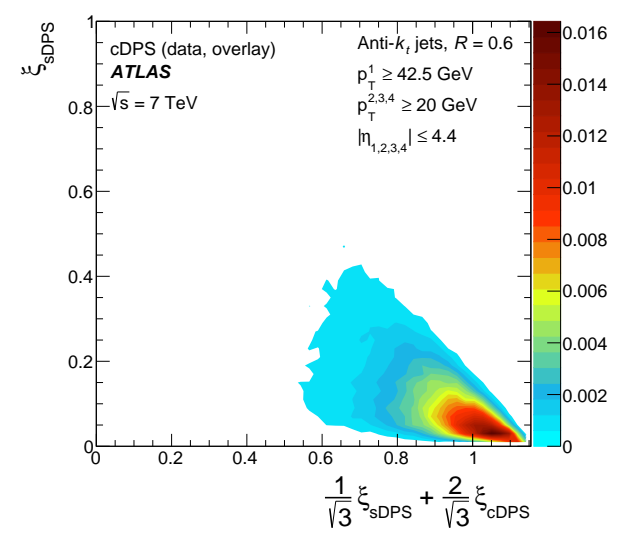

(b)

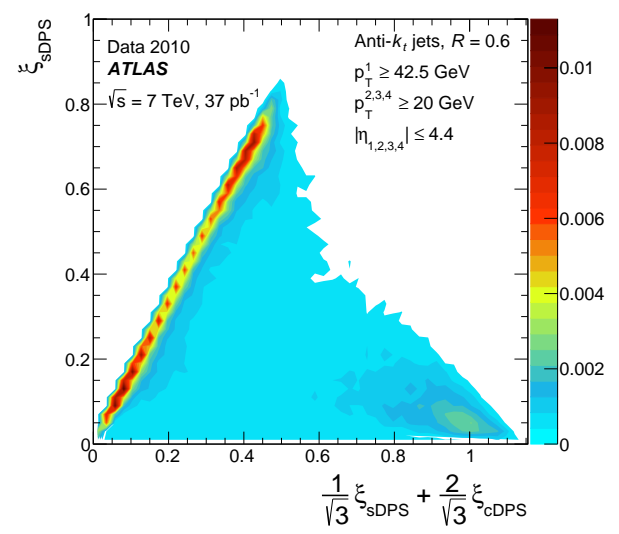

(d)

Figure 6: Normalized distributions of the NN outputs, mapped to a ternary plot as described in the text, in the (a) SPS, (b) cDPS, (c) sDPS test samples and (d) in the data [5].

fractions as free parameters:

$$
\mathscr{D}=\left(1-f_{c D P S}-f_{s D P S}\right) \mathscr{M}_{S P S}+f_{c D P S} \mathscr{M}_{c D P S}+f_{s D P S} \mathscr{M}_{s D P S} .
$$

The result of the $\chi^{2}$ fit is $f_{D P S}=0.092_{-0.011}^{+0.005}$ (stat. $)_{-0.037}^{+0.033}$ (syst.), and finally

$$
\left.\sigma_{\text {eff }}=14.9_{-1.0}^{+1.2} \text { (stat. }\right)_{-3.8}^{+5.1} \text { (syst.) mb. }
$$

The systematic errors are much larger than the statistical ones. A number of systematic uncertainties were taken into account. But the dominant contribution $\left({ }_{-19}^{+31} \%\right)$ is due to the jet energy scale uncertainty, which is about $4.5 \%$ in the central region, rising to about $10 \%$ in the forward region.

\section{Conclusions}

Primary-charged-particle multiplicity measurements with the ATLAS detector using proton- 
proton collisions delivered by the LHC at $\sqrt{s}=13 \mathrm{TeV}$ are presented. The results highlight clear differences between MC models and the measured distributions. Among the models considered EPOS reproduces the data the best, PYTHIA8 A2 and Monash give reasonable descriptions of the data and QGSJET-II provides the worst description of the data.

Several distributions sensitive to properties of the underlying event have been presented. They were measured by the ATLAS experiment at the LHC using low-luminosity $13 \mathrm{TeV}$ pp collision events. The measured observables are defined using charged tracks and have been corrected to the level of primary charged particles. An increase in UE activity of approximately $20 \%$ is observed when going from $7 \mathrm{TeV}$ to $13 \mathrm{TeV}$ pp collisions. Comparisons against predictions from several commonly used MC generator configurations indicate that for most observables the models describe the underlying-event data to better than 5\% accuracy. But this level of variation is far greater than the experimental uncertainty of the measurements, and there is evidence of systematic mismodelling.

A measurement of the total cross section from elastic pp scattering at $\sqrt{s}=8 \mathrm{TeV}$ was performed. The measurement is based on $500 \mu \mathrm{b}^{-1}$ of collision data collected in a high- $\beta^{*}$ run at the LHC in 2012 with the ALFA Roman Pot sub-detector. The optical theorem is used to extract the total cross section from the differential elastic cross section by extrapolating to $t \rightarrow 0$. The total cross section at $\sqrt{s}=8 \mathrm{TeV}$ is determined to be

$$
\left.\sigma_{t o t}(p p \rightarrow X)=96.07 \pm 0.18 \text { (stat. }\right) \pm 0.85 \text { (exp.) } \pm 0.31 \text { (extr.) mb. }
$$

A measurement of the rate of hard double-parton scattering in four-jet events was performed using a sample of data collected with the ATLAS experiment in 2010. It corresponds to an integrated luminosity of $37.3 \pm 1.3 \mathrm{pb}^{-1}$ of pp collisions at $\sqrt{s}=7 \mathrm{TeV}$. Finally the effective DPS cross-section is determined to be

$$
\sigma_{e f f}=14.9_{-1.0}^{+1.2}(\text { stat. })_{-3.8}^{+5.1}(\text { syst. }) \mathrm{mb} .
$$

\section{References}

[1] ATLAS Collaboration, The ATLAS Experiment at the CERN Large Hadron Collider, JINST 3 (2008) S08003.

[2] ATLAS Collaboration, Charged-particle distributions in $\sqrt{s}=13 \mathrm{TeV}$ pp interactions measured with the ATLAS detector at the LHC, Phys. Lett. B 758 (2016) 67, [arXiv:1602.01633].

[3] ATLAS Collaboration, Measurement of charged-particle distributions sensitive to the underlying event in $\sqrt{s}=13$ TeV proton-proton collisions with the ATLAS detector at the LHC, arXiv: 1701.05390.

[4] ATLAS Collaboration, Measurement of the total cross section from elastic scattering in pp collisions at $\sqrt{s}=8 \mathrm{TeV}$ with the ATLAS detector, Phys. Lett. B 761 (2016) 158, [arXiv: 1607.06605 ].

[5] ATLAS Collaboration, Study of hard double-parton scattering in four-jet events in pp collisions at $\sqrt{s}=7 \mathrm{TeV}$ with the ATLAS experiment, JHEP 11 (2016) 110, [arXiv: 1608.01857 ].

[6] ATLAS Collaboration, Measurement of the total cross section from elastic scattering in pp collisions at $\sqrt{s}=7$ TeV with the ATLAS detector, Nucl. Phys. B 889 (2014) 486, [arXiv: 1408 . 5778]. 